\title{
CRITÉRIO DE SELEÇÃO DO MOMENTO MAIS PROPÍCIO PARA INSPEÇÃO TERMOGRÁFICA DE FACHADAS COM REVESTIMENTO CERÂMICO ATRAVÉS DA ANÁLISE DA VARIAÇÃO DA TEMPERATURA MÉDIA DA REGIÃo.
}

\author{
AIDAR, LUIZ \\ Engenheiro Civil, Mestre \\ Universidade de Brasília \\ Distrito Federal; Brasil \\ luiz.augusto_aidar@hotmail.com
}

\author{
BAUER, ELTON \\ Engenheiro Civil, Doutor, Professor \\ Universidade de Brasília \\ Distriti Federal; Brasil \\ elbauerlem@gmail.com
}

\section{RESUMO}

No campo da construção civil, a termografia infravermelha pode ser utilizada como ferramenta para a inspeção de elementos da construção como, por exemplo, na inspeção de fachadas. Um dos grandes desafios do uso dessa tecnologia como ferramenta para inspeção de fachadas de edifícios é a seleção do momento mais propício para se analisar o termograma. Com isso, o presente estudo objetiva analisar séries de termogramas de descolamentos cerâmicos em diferentes momentos de inspeção a fim de se constatar o momento de maior contraste termográfico da anomalia. Os resultados indicam que as inspeções realizadas durante o período da manhã apresentam maior contraste termográfico quando comparadas às inspeções noturnas e que, dentre as inspeções da manhã, o contraste térmico tente a ser máximo após a máxima variação da temperatura média da região.

Palavras-chave: termografia, momento de inspeção, fachadas, revestimento cerâmico.

\section{ABSTRACT}

In the field of construction, infrared thermography can be used as a tool for inspecting building elements such as for façade inspection. One of the major challenges of using this technology, such as the building inspection tool of buildings, is selecting the most appropriate time to analyze the thermogram. Thus, this study aims to analyze the series of ceramic detachment thermometers at different times of inspection until the end of the period or the longest thermography period of the anomaly. The results that were performed during the morning occurred a longer period of contrast when compared to the nightly observations and that, among the morning occurrences, the thermal contrast tries to be the maximum after the average temperature of the region.

Keywords: thermography, inspection moment, facades, ceramic coating.

\section{INTRODUÇÃO}

O edifício deve manter seu desempenho durante a vida útil prevista em projeto sem os sinais de desgaste, desde que realizadas as intervenções periódicas de manutenção e conservação (ABNT, 2008; FERRAZ et al., 2013). Entretanto, todo elemento construtivo sofre processo de degradação (AIDAR, 2019). Esse pode ser mais ou menos intenso dependendo das condições de exposição, da sensibilidade à degradação de cada elemento e das intervenções de manutenção, sendo elas preditivas ou corretivas (BAUER; SILVA; ZANONI, 2015).

A constatação, identificação e mensuração de anomalias é essencial para que se possa avaliar a real situação do edifício e, desta forma, o levantamento das anomalias auxilia na proposição de soluções adequadas, sobretudo com foco no desempenho (DE FREITAS, 2012). Neste contexto, destaca-se a importância das inspeções que, se feitas no tempo correto são extremamente úteis. As anomalias podem ser identificadas precocemente e receberem o tratamento adequado. Caso a identificação seja feita tardiamente, a gravidade possivelmente será maior e consequentemente o custo de reparo será de maior valor (NASCIMENTO, 2016).

A fachada dos edifícios acumula as funções de proteger os espaços internos das condições adversas do exterior, proporcionar conforto higrotérmico e acústico e segurança às ações excepcionais (CONSOLI, 2006). Até por isso, as fachadas caracterizam-se por ser um dos principais condicionantes no desempenho de um edifício (SILVA, 2014). A 
fachada é suscetível à degradação mais intensa, devido ao fato de estar em contato direto com diversos agentes de degradação, como os agentes atmosféricos, radiação solar, chuva dirigida e temperatura (BAUER et al., 2015). Assim o risco de falhas no desempenho se torna importante principalmente quando existe alta exposição aos agentes de degradação climáticos (AIDAR, 2019).

Existem diversas técnicas de inspeção, as quais podem ser classificadas em destrutivas e não destrutivas. As inspeções destrutivas caracterizam-se por afetarem objeto de estudo como o carregamento externo de estruturas de concreto para mensuração da resistência dos elementos. Já os ensaios não destrutivos não afetam o objeto como ocorre no ensaio de precurssão para detecção de descolamentos cerâmicos (AIDAR, 2019).

$\mathrm{Na}$ detecção de anomalias de fachadas de edifícios, a termografia destaca-se por permitir a obtenção de resultados a distância e em tempo real, o que possibilita análise da qualidade dos resultados durante a obtenção desses, gerando como resultados termogramas que são representações no plano das temperaturas superficiais do objeto de estudo (BAUER et al., 2016; EDIS; FLORES-COLEN; DE BRITO, 2014, 2015; FOX et al., 2015).

Dentre as patologias que podem ser detectadas em fachadas com o uso da termografia, destacam-se a presença de umidade, de vazios, de materiais ocultos, de fissuras e de descolamentos de placas cerâmicas, como indicado na Figura 1 (AIDAR, 2019). Cabe ressaltar que a detecção dessas anomalias com o uso da termografia está intrinsecamente ligada à experiência do termografista e às condições de obtenção das imagens, sendo que não existem procedimentos padrões, critérios ou normas ligados à aplicação dessa técnica (PAVÓN, 2017).

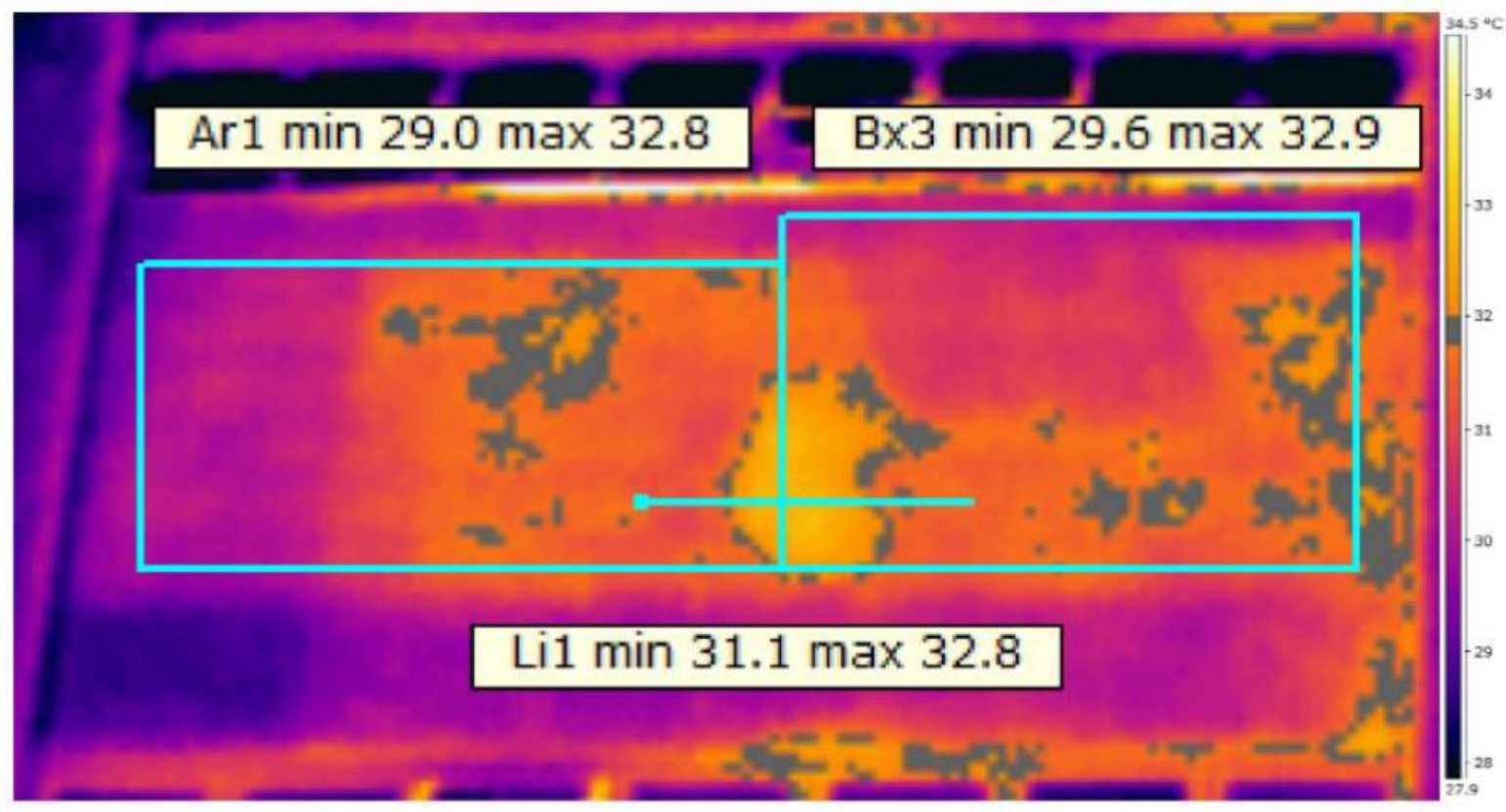

Figura 1 - Detecção de descolamento cerâmico com uso de termografia.

O presente estudo objetiva analisar séries de termogramas de descolamentos cerâmicos em diferentes momentos de inspeção a fim de se constatar o momento de maior contraste termográfico da anomalia. Dessa forma, busca-se padronizar horários mais propícios à constatação de anomalias durante a realização de inspeções termográficas.

\section{METODOLOGIA}

A metodologia para se estudar o momento mais propício para a realização da inspeção predial em fachadas de edifícios com revestimento cerâmico foi a comparação dos valores do Delta-T (equação 1) para diferentes momentos do dia.

$$
\text { Delta- } \mathrm{T}=\mathrm{Ta}-\mathrm{Tn}
$$

Onde: $\mathrm{Ta}=$ temperatura da região com anomalia

Tn = temperatura da região sem anomalia 
O cálculo do Delta-T é feito a partir da exportação de linhas traçadas no software QuickReport para planilhas Excel. Inicialmente, é traçada uma linha que percorra toda a anomalia a ser analisada. É importante destacar que a posição dessa linha é mantida o mais uniforme possível nas diferentes inspeções realizadas, a fim de se manter os pontos de análise. Para se manter a posição da linha, essa foi colocada em relação a algum elemento fixo como janelas e limites da fachada.

O valor de Delta-T é o máximo valor entre o Delta-T de entrada e o de saída. Delta-T de entrada é definido como a subtração entre o máximo valor de temperatura interna do defeito e o pixel anterior à anomalia. Já o de saída é dado pela subtração entre o máximo valor de temperatura interna do defeito, idem ao usado no Delta-T de entrada e o pixel posterior à anomalia. Para exemplificar o processo é apresentado o exemplo na Tabela 5.

Tabela 1 - Exemplo de determinação do Delta-T.

\begin{tabular}{|c|c|c|c|c|c|c|c|}
\hline Pixel & 6 & 7 & 8 & 9 & 10 & 11 & 12 \\
\hline Temperatura $\left({ }^{\circ} \mathrm{C}\right)$ & 24,54 & 25,12 & 25,34 & 25,23 & 25,06 & 25,12 & 25,04 \\
\hline Média & 24,83 & 25,23 & 25,29 & 25,15 & 25,09 & 25,08 & 24,83 \\
\hline Variação & 0,4 & 0,06 & $-0,14$ & $-0,06$ & $-0,01$ & $-0,25$ & $-0,35$ \\
\hline Temperatura máxima dentro da anomalia $\left({ }^{\circ} \mathrm{C}\right)$ & \multicolumn{7}{|c|}{25,34} \\
\hline Temperatura antes de entrar na anomalia $\left({ }^{\circ} \mathrm{C}\right)$ & \multicolumn{7}{|c|}{24,54} \\
\hline Temperatura após sair da anomalia $\left({ }^{\circ} \mathrm{C}\right)$ & \multicolumn{7}{|c|}{25,04} \\
\hline Delta-T de entrada $\left({ }^{\circ} \mathrm{C}\right)$ & \multicolumn{7}{|c|}{0,8} \\
\hline Delta-T de saída $\left({ }^{\circ} \mathrm{C}\right)$ & \multicolumn{7}{|c|}{0,3} \\
\hline Delta-T $\left({ }^{\circ} \mathrm{C}\right)$ & \multicolumn{7}{|c|}{0,8} \\
\hline
\end{tabular}

Foram estudados 4 blocos residenciais localizados na Asa Norte - Brasília, todos eles apresentam revestimento em placas cerâmica, sendo que apenas o Edifícios D também apresenta áreas com revestimento em argamassa. Tais áreas em argamassa não foram analisadas no estudo. A Tabela 2 apresenta todas as pastilhas cerâmicas usadas nas fachadas nos edifícios com suas respectivas cores e absortâncias. Além disso, indica-se a emissividade utilizada no estudo, essa foi tomada como a mesma para todos os edifícios por se tratar de um mesmo material.

Tabela 2 - Tonalidade dos revestimentos.

\begin{tabular}{c|c|c|c}
\hline Edifício & Pastilha & Cor da pastilha & 0,787 \\
\hline \multirow{2}{*}{ A } & & Verde-musgo & 0,264 \\
\cline { 2 - 4 } & & Verde erva-doce & 0,282 \\
\hline \multirow{2}{*}{ B e C } & & Branco & 0,706 \\
\hline \multirow{2}{*}{ D } & & Telha & 0,282 \\
\cline { 2 - 4 } & & Branco & \\
\hline \multicolumn{2}{c|}{ Todos } & Emissividade & \\
\hline
\end{tabular}

Os edifícios estão localizados na zona bioclimática 4 conforme classificação do zoneamento bioclimático brasileiro recomendado pela NBR 15220-3 Desempenho térmico de edificações - Parte 3: Zoneamento bioclimático brasileiro e diretrizes construtivas para habitações unifamiliares de interesse social (ABNT, 2015). Além disso, todos os edifícios apresentam estrutura composta de vigas e pilares de concreto e bloco cerâmico como vedação.

Os horários de inspeção foram pré-selecionados através da análise dos dados oriundos da simulação higrotérmica feita com uso do software WUFI Pro 5.3. Dentre os diversos dados obtidos a partir da simulação, a precipitação e a temperatura nas diferentes camadas do revestimento foram utilizadas para análise do momento mais propício a se inspecionar o edifício. 
A precipitação foi utilizada para se estudar dias em que a umidade proveniente da chuva não afetasse as imagens. Para isso foram tomados dias que apresentassem um período anterior sem precipitação de, pelo menos, dois dias. Cabe ressaltar que foram selecionados os dias próximos aos dias das inspeções para que não houvesse discrepância quanto aos dados.

Quanto à temperatura das camadas do revestimento, essas foram utilizadas para o cálculo do gradiente superfície- bloco (PAVÓN, 2017), o qual foi utilizado para escolha dos horários de inspeção. Os dados gerados na simulação para o dia característico escolhido são analisados segundo os parâmetros para todas as orientações de fachadas a serem inspecionadas:

- Momento do máximo valor de gradiente GSpBc, para análise no fluxo direto;

- Período de tempo com gradiente GSpBc crescente, para análise no fluxo direto;

- Momento do mínimo valor de gradiente GSpBc, para análise no fluxo inverso;

- Período de tempo com gradiente GSpBc decrescente, para análise no fluxo inverso;

- Período de tempo com gradiente GSpBc superior a 0,6, como indicado por Pavón (2017);

Durante a inspeção predial, obtêm-se os valores de temperatura ambiente, umidade relativa do ar e temperatura refletida para cada sessão de obtenção das imagens. Esse parâmetro deve ser obtido e inserido na câmera a cada nova sequência de imagens, objetivando menores desvios devido a essas variáveis. Para os dados de temperatura ambiente e umidade relativa utiliza-se o psicrômetro Extech MO297 com termômetro acoplado; a temperatura refletida foi medida através do método do papel alumínio como descrito na ASTM E1862 (ASTM 1862, 2014);

Para obtenção dos termogramas utilizou-se a câmera FLIR T400 com faixa de temperatura entre $-20^{\circ} \mathrm{C}$ e $120^{\circ} \mathrm{C}$, precisão de $2 \%$, faixa espectral de 7,5 a $13 \mu \mathrm{m}$, resolução de 320 x 240 pixels, lente FOL $18 \mathrm{~mm}$ e IFOV (lente de $25^{\circ}$ ) $1,36 \mathrm{mRad}$ para a primeira inspeção e lente de $15^{\circ}$ com IFOV 0,820 nas demais inspeções. A troca da lente durante a realização da pesquisa se justifica pelas distâncias entre os pontos de inspeção e as fachadas, haja vista que a lente adotada a partir da segunda inspeção possui IFOV menor.

Após a obtenção dos termogramas, esses são tratados e estudados a partir do uso do software QuickReport 1.2. Nesse, as fachadas dos edifícios são dividias em pequenas áreas que são analisadas isoladamente para facilitação da detecção das anomalias e aumento da precisão das medidas. Detectadas as anomalias, essas são estudadas quantitativamente através do cálculo do Delta-T.

\section{RESULTADOS}

Após análise dos dados obtidos com a simulação higrotérmica foram definidos dois grupos de momentos de inspeção. O primeiro a ser realizado com fluxo direto pela manhã e o segundo no final da tarde e início da noite. Os momentos de inspeção estão indicados na Tabela 3. Cabe aqui apresentar algumas ressalvas quanto às inspeções para que o entendimento dos dados e de sua análise não seja dificultado. Inicialmente, constata-se que os horários de inspeção variam quando se comparam as inspeções pela manhã do Edifício A com as demais. Isso ocorreu, pois o Edifício A foi tomado como estudo preliminar e, após sua análise, houve dúvidas a respeito do comportamento da fachada em horários anteriores às 7:40, Além disso, às 11:00 foi relatado pequena precipitação na região, o que poderia afetar as imagens.

Tabela 3- Horários de inspeção.

\begin{tabular}{c|c|c}
\hline Grupo & Edifício A & Edifícios B, C e D \\
\hline \multirow{4}{*}{ Manhã } & - & $07: 00$ \\
\cline { 2 - 3 } & $07: 40$ & $08: 00$ \\
\cline { 2 - 3 } & $08: 30$ & $09: 00$ \\
\cline { 2 - 3 } & $09: 30$ & $10: 00$ \\
\cline { 2 - 3 } & $10: 30$ & $11: 00$ \\
\hline \multirow{4}{*}{ Noite } & - & $12: 00$ \\
\cline { 2 - 3 } & $16: 50$ & - \\
\cline { 2 - 3 } & $17: 30$ & $17: 40$ \\
\cline { 2 - 3 } & $18: 20$ & $18: 30$ \\
\cline { 2 - 3 } & $19: 10$ & $19: 20$ \\
\hline
\end{tabular}


Comparando-se inicialmente apenas o contraste termográfico em relação ao fluxo térmico, verificou-se que os valores de Delta-T apresentavam valores superiores nas inspeções realizadas durante a manhã, quando comparados com os valores referentes às inspeções da noite, como indicado na FIG. Foram analisados 163 casos de descolamentos cerâmicos, dentre esses, apenas 6 apresentaram contraste termográfico superior durante a noite. Esses resultados indicam que o momento mais propício para se fazer uma inspeção termográfica é durante a manhã.

Após conclusão de que o período da manhã apresenta melhores resultados de Delta-T em comparação com o período da noite, fez-se a análise mais detalhada do contraste das anomalias durante as inspeções pela manhã como objetivo de refinar os momentos em que as inspeções devem ser realizadas.

Os dados de contraste termográfico foram estudados em relação a várias variáveis independentes. Dentre essas, a variação da temperatura média da região na qual a anomalia estava presente apresentou comportamento notável. A Tabela 4 indica os valores relativos a alguns casos da fachada norte do edifício A.

Tabela 4 - Delta-T de descolamento cerâmico e Variação da temperatura média da região - Fachada Norte (Edifíco A).

\begin{tabular}{|c|c|c|c|}
\hline Região & Momento da Inspeção & $\begin{array}{c}\text { Variação da } \\
\text { temperatura média } \\
\left({ }^{\circ} \mathrm{C}\right)\end{array}$ & Delta-T $\left({ }^{\circ} \mathrm{C}\right)$ \\
\hline \multirow{4}{*}{ A6 } & $07: 30$ & - & 0,19 \\
\hline & $08: 40$ & 1,10 & 0,42 \\
\hline & 09:40 & 0,40 & 0,46 \\
\hline & $10: 40$ & 3,50 & 0,66 \\
\hline \multirow{4}{*}{ D4 } & $07: 30$ & - & 0,41 \\
\hline & 08:40 & 2,00 & 1,04 \\
\hline & 09:40 & 0,80 & 0,71 \\
\hline & $10: 40$ & 4,40 & 1,28 \\
\hline \multirow{4}{*}{ G1 } & $07: 30$ & - & 0,16 \\
\hline & 08:40 & 1,60 & 0,44 \\
\hline & 09:40 & 0,70 & 0,43 \\
\hline & $10: 40$ & 2,10 & 0,63 \\
\hline
\end{tabular}

Constata-se que o Delta-T máximo foi encontrado na inspeção realizada às 10:40, a qual apresenta a maior variação de temperatura média em relação a inspeção anterior. O comportamento referido também é verificado em todas as orientações de fachadas para todos os edifícios estudados como indicado na Tabela 5, para a fachada sul do Edifício B; na Tabela 6 para a fachada leste do edifício C; e na Tabela 7 para a fachada Oeste do Edifício D. 
Tabela 5 - Delta-T de descolamento cerâmico e Variação da temperatura média da região - Fachada Sul (Edifíco B).

\begin{tabular}{|c|c|c|c|}
\hline Região & Momento da Inspeção & $\begin{array}{c}\text { Variação da } \\
\text { temperatura média } \\
\left({ }^{\circ} \mathrm{C}\right)\end{array}$ & Delta-T $\left({ }^{\circ} \mathrm{C}\right)$ \\
\hline \multirow{6}{*}{ B1 } & 07:00 & - & 0,25 \\
\hline & 08:00 & 0,20 & 0,49 \\
\hline & 09:00 & 1,20 & 0,60 \\
\hline & $10: 00$ & 1,60 & 0,99 \\
\hline & 11:00 & 0,80 & 0,60 \\
\hline & $12: 00$ & 0,80 & 0,55 \\
\hline \multirow{6}{*}{$\mathrm{C} 3$} & 07:00 & - & 0,72 \\
\hline & 08:00 & 0,00 & 0,78 \\
\hline & 09:00 & 1,00 & 1,00 \\
\hline & $10: 00$ & 1,90 & 1,26 \\
\hline & 11:00 & 0,50 & 0,82 \\
\hline & $12: 00$ & 1,30 & 1,09 \\
\hline \multirow{6}{*}{$\mathrm{F} 2$} & 07:00 & - & 0,52 \\
\hline & 08:00 & 0,30 & 0,50 \\
\hline & 09:00 & 0,70 & 0,55 \\
\hline & $10: 00$ & 2,10 & 0,73 \\
\hline & 11:00 & 0,60 & 0,52 \\
\hline & $12: 00$ & 1,50 & 0,68 \\
\hline
\end{tabular}

Tabela 6 - Delta-T de descolamento cerâmico e Variação da temperatura média da região - Fachada Leste (Edifício C).

\begin{tabular}{|c|c|c|c|}
\hline Região & Momento da Inspeção & $\begin{array}{c}\text { Variação da } \\
\text { temperatura média } \\
\left({ }^{\circ} \mathrm{C}\right)\end{array}$ & Delta-T $\left({ }^{\circ} \mathrm{C}\right)$ \\
\hline \multirow{6}{*}{ B1 } & 07:00 & - & 0,61 \\
\hline & 08:00 & 9,60 & 0,98 \\
\hline & 09:00 & 6,00 & 0,86 \\
\hline & $10: 00$ & 1,60 & 0,40 \\
\hline & 11:00 & $-1,40$ & 0,55 \\
\hline & $12: 00$ & $-2,40$ & 0,72 \\
\hline \multirow{6}{*}{ E1 } & 07:00 & - & 0,25 \\
\hline & 08:00 & 1,10 & 0,39 \\
\hline & 09:00 & 8,90 & 1,77 \\
\hline & 10:00 & 5,30 & 1,25 \\
\hline & 11:00 & 0,20 & 0,43 \\
\hline & $12: 00$ & $-2,40$ & 0,31 \\
\hline \multirow{6}{*}{$\mathrm{F} 1$} & 07:00 & - & 0,20 \\
\hline & 08:00 & 0,70 & 0,10 \\
\hline & 09:00 & 8,50 & 1,11 \\
\hline & $10: 00$ & 5,90 & 0,43 \\
\hline & 11:00 & 0,60 & 0,41 \\
\hline & $12: 00$ & $-2,30$ & 0,12 \\
\hline
\end{tabular}


Tabela 7 - Delta-T de descolamento cerâmico x Variação da temperatura média da região - Fachada Oeste (Edifício D).

\begin{tabular}{c|c|c|c}
\hline \multirow{4}{*}{ Região } & Momento da Inspeção & $\begin{array}{c}\text { Variação da } \\
\text { temperatura média } \\
\left({ }^{\circ} \mathrm{C}\right)\end{array}$ & Delta-T $\left({ }^{\circ} \mathrm{C}\right)$ \\
\hline \multirow{5}{*}{ B1 } & $07: 00$ & - & 0,74 \\
\cline { 2 - 4 } & $08: 00$ & 0,00 & 0,64 \\
\cline { 2 - 4 } & $09: 00$ & 0,90 & 0,89 \\
\cline { 2 - 4 } & $10: 00$ & 1,90 & 1,19 \\
\cline { 2 - 4 } & $11: 00$ & 1,50 & 0,58 \\
\hline \multirow{5}{*}{$\mathrm{C} 1$} & $12: 00$ & 0,60 & 0,58 \\
\hline & $07: 00$ & - & 0,58 \\
\cline { 2 - 4 } & $08: 00$ & 0,00 & 0,68 \\
\cline { 2 - 4 } & $09: 00$ & 0,80 & 0,65 \\
\cline { 2 - 4 } & $10: 00$ & 1,20 & 1,18 \\
\hline & $11: 00$ & 1,60 & 0,48 \\
\hline
\end{tabular}

A Tabela 8 apresenta ainda o momento com maior valor de GSpBc para cada fachada inspecionada. Os dados indicam proximidade entre os momentos mais propícios para a realização das inspeções através das duas análises. Isso indica que a análise do gradiente GSpBc é válida para a seleção dos momentos de inspeção.

Tabela 8 - Momento mais propício para inspeção termográfica por fachada.

\begin{tabular}{c|c|c|c}
\hline \multirow{4}{*}{ Fachada } & Edifício & $\begin{array}{c}\text { Momento com maior } \\
\text { variação de temperatura } \\
\text { média da região }\end{array}$ & $\begin{array}{c}\text { Momento com maior valor } \\
\text { de GSpBc }\end{array}$ \\
\hline \multirow{3}{*}{ Norte } & A & $10: 40$ & $10: 00$ \\
\cline { 2 - 4 } & B & $10: 00$ e 11:00 & $10: 00$ \\
\cline { 2 - 4 } & D & $10: 00$ e 11:00 & $10: 00$ \\
\hline \multirow{2}{*}{ Sul } & A & $10: 40$ & $09: 00$ \\
\hline \multirow{2}{*}{ Leste } & B & $10: 00$ & $09: 00$ \\
\cline { 2 - 4 } & A & $08: 30$ & $09: 00$ \\
\cline { 2 - 4 } & $\mathrm{C}$ & $08: 00$ e 09:00 & $10: 00$ \\
\hline \multirow{2}{*}{ Oeste } & $\mathrm{D}$ & $08: 00$ & $09: 00$ \\
\hline
\end{tabular}

\section{CONCLUSÕES}

Os resultados demonstram que as anomalias de descolamento cerâmico, independentemente da orientação da fachada e da cor da pastilha, são melhor detectados quando há uma grande variação da temperatura média da região onde a anomalia se localiza. Isso se justifica, pois a diferença de resposta entre as áreas com e sem anomalia será maior com a maior mais intensa do fluxo.

Quanto à máxima variação da temperatura média das regiões, esse parâmetro apresenta comportamento semelhante quando comparado fachada por fachada dos diferentes edifícios. Esses dados indicam grande variabilidade dos horários de inspeção a depender da orientação da fachada, sendo que a fachada Leste apresenta maior variação de temperatura média entre 08:00 e 09:00, enquanto a oeste entre 10:00 e 11:00, o que vai de encontro a realização das inspeções entre 07:00 e 12:00 a fim de se abranger a inspeção de todas as fachadas. 


\section{REFERÊNCIAS}

ABNT. NBR 15575: Edifícios habitacionais de até cinco pavimentos - Desempenho. Parte 1: Requisitos geraisRio de Janeiro, 2008.

ABNT. NBR 15220-3 Desempenho térmico de edificações - Parte 3: Zoneamento bioclimático brasileiro e diretrizes construtivas para habitações unifamiliares de interesse social., 2015.

AIDAR, L. Metodologia de inspeção e análise de termogramas para estudo de fachadas em revestimento cerâmico. [s.l.] Universidade de Brasília, 2019.

ASTM 1862. Standard Practice for Measuring and Compensating for Reflected Temperature Using Infrared Imaging Radiometers, 2014.

BAUER, E. et al. Infrared thermography - evaluation of the results reproducibility. Structural Survey, v. 33, p. 20-35, 2015 .

BAUER, E. et al. Criteria for Identification of Ceramic Detachments in Building Facades with Infrared ThermographyRecent Developments in Building Diagnosis Techniques, 2016. Disponível em: <http://link.springer.com/10.1007/978-981-10-0466-7>

BAUER, E.; SILVA, M. N. B.; ZANONI, V. A. G. Mensuração da degradação e vida útil em fachadas. XI Simpósio Brasileiro de Tecnologia das Argamassas, n. April, 2015.

CONSOLI, O. J. Análise da durabilidade dos componentes das fachadas de edifícios, sob a ótica do projeto arquitetônicoFlorianópolisPrograma de Pós-Graduação da Faculdade em Engenharia civil da Universidade Federal de Santa Catarina, , 2006.

DE FREITAS, V. P. Manual de apoio ao projecto de reabilitação de edifícios antigos. Lisboa - Portugal: [s.n.]. EDIS, E.; FLORES-COLEN, I.; DE BRITO, J. Time-dependent passive infrared thermographic inspection of facades. XIII International Conference on Durability of Building Materials and Componentes - XIII DBMC, n. November $2015,2014$.

EDIS, E.; FLORES-COLEN, I.; DE BRITO, J. Quasi-quantitative infrared thermographic detection of moisture variation in facades with adhered ceramic cladding using principal component analysis. Building and Environment, v. 94, n. P1, p. 97-108, 2015.

FERRAZ, G. T. et al. State-of-the-Art Review of Building Inspection Systems. Journal of Performance of Constructed Facilities, p. 1-8, 2013.

FOX, M. et al. Time-lapse thermography for building defect detection. Energy and Buildings, v. 92, p. 95-106, 2015. NASCIMENTO, M. Aplicação da simulação higrotérmica na investigação da degradação de fachadas de edifíciosBrasília- BrazilUniversidade de Brasília (UnB), , 2016. Disponível em: <http://www.pecc.unb.br/wpcontent/uploads/dissertacoes/M16-18A-Matheus-Nascimento.pdf>

PAVÓN, E. Critérios e padrões de comportamento para avaliação de descolamentos cerâmicos com termografia de infravermelho.Brasília- BrazilUniversidade de Brasília (UnB), , 2017.

SILVA, M. N. B. Avaliação Quantiativa da Degradação e Vida ùtil de Revestimentos de Fachada - Aplicação ao Caso de BrasíliaUniversidade de Brasília (UnB), , 2014. 